\title{
Factors Related to the Success of Endovascular Therapy with Plain Old Balloon Angioplasty of Central Vein Stenosis in Haemodialysis Patients Akhmadu Muradia*, Donie Firdhianto ${ }^{b}$, Aria Kekalih ${ }^{c}$
}

Introduction: Central venous stenosis (CVS) or occlusion is a severe complication in hemodialysis patients, which significantly decreases the patency of all vascular dialysis access components, including arteries and branches, AV anastomosis, peripheral veins, and central veins. The main etiology of CVS is mostly secondary to the placement of temporary or permanent dialysis catheters in the subclavian vein, internal jugular vein, and femoral vein. Standard endovascular therapy for central venous stenosis is conventional balloon angioplasty.

Method: This is a retrospective study using medical records from June 2013 to August 2018. Patients who underwent plain old balloon angioplasty (POBA) procedures in the CVS condition due to the installation of hemodialysis catheter access were included in this study. The analysis was performed to assess the characteristics and data distribution of each variable.

Results: Significant factors related to the success of endovascular procedure in patients with central venous stenosis with POBA were the onset of clinical symptoms ( $<3$ months; $p$ $<0.001$ ), duration of catheter placement ( $<2.5$ months; $p<0.001)$, history of previous catheter placement (no more than once, $p<0.001$ ), initial stenosis $(<80 ; p<0.001$ ), and diameter of POBA ( $\geq 10 \mathrm{~mm} ; \mathrm{p}<0.001$ ).

Conclusion: Some factors influenced the success of the POBA procedure for overcoming CVS. The need to understanding the use of hemodialysis catheter access according to the guideline is important.

Keywords: central venous stenosis, endovascular therapy, plain old balloon angioplasty https://doi.org/10.36864/jinasvs.2020.1.002
Copyright (C) 2020, The Indonesian Society for Vascular and Endovascular Surgery

JINASVS 2020;1(1):6-9

*Correspondence: akhmadumuradi@gmail.com

aM.D., Ph.D., Vascular and Endovascular Division, Department of Surgery, Cipto Mangunkusumo Hospital - Faculty of Medicine, Universitas Indonesia, Jakarta, Indonesia

${ }^{b}$ M.D., Department of Surgery, Semarang Medical Center Hospital Telogorejo, Semarang, Indonesia

cM.D., M.T.I., Ph.D., Department of Community Medicine, Faculty of Medicine Universitas Indonesia, Jakarta, Indonesia

\section{INTRODUCTION}

Central venous stenosis or occlusion is a severe complication in hemodialysis patients, which significantly decreases the quality of effective hemodialysis. The main etiology of central venous stenosis (CVS) is mostly secondary to the placement of transient or permanent dialysis catheters. The prevalence of venous stenosis or thrombosis after the use of hemodialysis catheters ranges from 10 to $50 \% .^{1.2}$ Central venous stenosis is far more frequent in subclavian venous catheterization than in internal jugular veins. ${ }^{3}$

The most common symptoms of CVS are swelling of the arm, and partial swelling in the ipsilateral face, difficulty in cannulation of venous arms during hemodialysis (in the use of arteriovenous fistulas), and can cause increased recirculation. This also impacts on the placement of a temporary or permanent central venous catheter in the future. ${ }^{4}$
The standard endovascular therapy for central venous stenosis is plain old balloon angioplasty (POBA). The reported primary patency rates of this technique at six months and 12 months were $45 \pm 8 \%$ and $29 \pm 8 \% .^{2}$ Stenting is indicated in cases of elastic CVS or recurrent stenosis over three months, according to the National Kidney Foundation Initiative Disease Outcomes Initiative (NKF-KDOQI) guideline. However, stenting is not suitable in some cases because of the position of the stenosis, especially near the central venous bifurcation, including the branching of the right and left brachiocephalic veins and the branching sites of the subclavian and internal jugular veins. Early intrastent restenosis is also a frequent problem for shunt dysfunction, venous hypertension, and ipsilateral arm edema. ${ }^{2,3,5}$

This study aims to determine the factors that influence the success of actions on CVS after endovascular measures using POBA. 


\section{METHOD}

This was an analytical retrospective study conducted in the Division of Vascular and Endovascular Surgery, Cipto Mangunkusumo Hospital, Indonesia from June 2013 to August 2018. Patients diagnosed with upper central venous stenosis (subclavian vein, internal jugular vein, truncus vein brachiocephalic, innominate vein and superior vena cava) after installation of central venous access, and had a venoplasty procedure were included in this study. Central venous stenosis of the lower trunk of the iliac vein and inferior vena cava, swelling of the upper arm caused by etiologies other than deep venous abnormalities (lymphedema, neurofibroma, soft tissue tumors, and else), deep venous stenosis caused by other than the placement of $\mathrm{CDL}$ (such as severe inflammatory diseases), infection at the location of intravenous catheters, thoracic outlet syndrome, post-radiation fibrosis, mediastinal fibrosis, history of pacemaker installation, and malignancy, were excluded from this study.

The data about the outcome after the endovascular procedure, history of central venous catheter placement, catheter type, symptom onset, and duration of catheter placement were extracted. Venoplasty data of the location of the stenosis, percentage of initial stenosis, venoplasty success, defined as residual stenosis $<30 \%$, were taken from the procedure report. Balloons angioplasty used had diameters of 10 to $12 \mathrm{~mm}$, burst pressures of 8 to $15 \mathrm{~atm}$, and operational pressures of 10 to $20 \mathrm{~atm}$. Technical failure was defined as residual stenosis of more than $30 \%$ remaining at the end of the intervention. Early failure was defined as the inability to cross the lesion during the main procedure or in the presence of occlusion or $50 \%$ restenosis within the first 30 days after the initial procedure. Data processing was performed using SPSS 20.0 for Windows ${ }^{\circledR}$.

\section{RESULTS}

There were a total of 62 patients with central venous stenosis who performed endovascular procedure Cipto Mangunkusumo from June 2013 to August 2018. Male had a prevalence of $71 \%$, the median age of subjects was 57 years (range 23-78 years), and mostly catheter placement locations are in subclavian $(74.2 \%)$. The location of stenosis was more numerous in the subclavian vein $(51.6 \%)$ than in the innominate vein, and clinical symptom onset of more than three months was $53.2 \%$. The duration of catheter placement $<2.5$ months was $51.6 \%$, with previous catheterization history was $58 \%$. Initial stenosis for $>80 \%$ was as much as $59.7 \%$, previous arteriovenous fistula (AVF) history in a total of 59 patients (in which $72.6 \%$ AVF was brachiocephalic), and $61.3 \%$ of patients with comorbid hypertension but without diabetes mellitus. In a total of 49 patients who had venoplasty, obtained $81.6 \%$ data using a POBA balloon with a diameter of $>10 \mathrm{~mm}$ and $70.8 \%$ with a pressure of $>8 \mathrm{~atm}$ (table 1 ). The success rate of the endovascular procedure in this study was $79 \%$ (table 1 ).

From the analysis, it was found the factors related to the success of the endovascular procedure in patients with central venous stenosis (Table 2 ).
Table 1. Characteristics of subjects $(n=62)$.

\begin{tabular}{|c|c|c|c|}
\hline Characteristic & 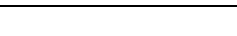 & $\mathbf{n}$ & $\%$ \\
\hline Location of & Jugular & 14 & $22.6 \%$ \\
\hline catheter & Subclavia & 46 & $74.2 \%$ \\
\hline & No catheter & 2 & $3.2 \%$ \\
\hline & Total & 62 & $100.0 \%$ \\
\hline Location of & Inominata & 30 & $48.4 \%$ \\
\hline central venous & Subclavia & 32 & $51.6 \%$ \\
\hline stenosis & Total & 62 & $100.0 \%$ \\
\hline Onset of clinical & $<3$ months & 29 & $46.8 \%$ \\
\hline symptoms & $\geq 3$ months & 33 & $53.2 \%$ \\
\hline & Total & 62 & $100.0 \%$ \\
\hline Duration of & $<2.5$ months & 32 & $51.6 \%$ \\
\hline catheter & $\geq 2.5$ months & 30 & $48.4 \%$ \\
\hline placement & Total & 62 & $100.0 \%$ \\
\hline History of & $\leq 1$ & 36 & $58.1 \%$ \\
\hline previous & $>1$ & 26 & $41.9 \%$ \\
\hline $\begin{array}{l}\text { dialysis } \\
\text { catheterization }\end{array}$ & Total & 62 & $100.0 \%$ \\
\hline Initial stenosis & $\leq 80 \%$ & 37 & $59.7 \%$ \\
\hline & $>80 \%$ & 25 & $40.3 \%$ \\
\hline & Total & 62 & $100 \%$ \\
\hline AVF position & Brachiocephalic & 45 & $72.6 \%$ \\
\hline & Radiocephalic & 14 & $22.6 \%$ \\
\hline & No AVF & 3 & $4.8 \%$ \\
\hline & Total & 62 & $100.0 \%$ \\
\hline Hypertension & With diabetes & 24 & $38.7 \%$ \\
\hline & $\begin{array}{l}\text { Without } \\
\text { diabetes }\end{array}$ & 38 & $61.3 \%$ \\
\hline & Total & 62 & $100.0 \%$ \\
\hline Performed & Success & 49 & $79 \%$ \\
\hline venoplasty & Failure & 13 & $21 \%$ \\
\hline & Total & 49 & $100 \%$ \\
\hline POBA diameter & $<10 \mathrm{~mm}$ & 9 & $18.4 \%$ \\
\hline & $\geq 10 \mathrm{~mm}$ & 40 & $81.6 \%$ \\
\hline & Total & 49 & $100.0 \%$ \\
\hline POBA pressure & $<8$ atm & 14 & $29.2 \%$ \\
\hline & $\geq 8$ atm & 34 & $70.8 \%$ \\
\hline & Total & 48 & $100 \%$ \\
\hline Catheter double & Temporary & 47 & $78 \%$ \\
\hline lumen type & Permanent & 13 & $22 \%$ \\
\hline
\end{tabular}

Only 31 patients provided data regarding central venous patency after the endovascular procedure. It was found that the median patency duration was six months, with a patency range of 212 months. Then after clinically suspected central venous restenosis, in 13 cases was performed with AVF closure surgeries, 8 with endovascular reinterventions using POBA with the unsuccessful technical outcome (residual stenosis $>30 \%$ ), and the remaining seven were not intervened.

\section{DISCUSSION}

From June 2013 to August 2018, endovascular procedures were performed in 62 patients with central venous stenosis, with male sex characteristics was $71 \%$, and the median age of 57 years (range 23 to 78 years). Most of the catheters were placed on the subclavian vein $(74.2 \%)$. This was unfortunate because, according to KDOQI 2006, the subclavian vein is the last choice for the location of installing hemodialysis access. The location of stenosis was more prominent in the subclavian vein $(51.6 \%)$ than in the innominate vein. This following the location prevalence of the catheter installation in this study, in which the majority were in the subclavian vein $(74.2 \%)$, triggers more twist, and 
Table 2. Factors related to the success of patency in central venous stenosis with POBA.

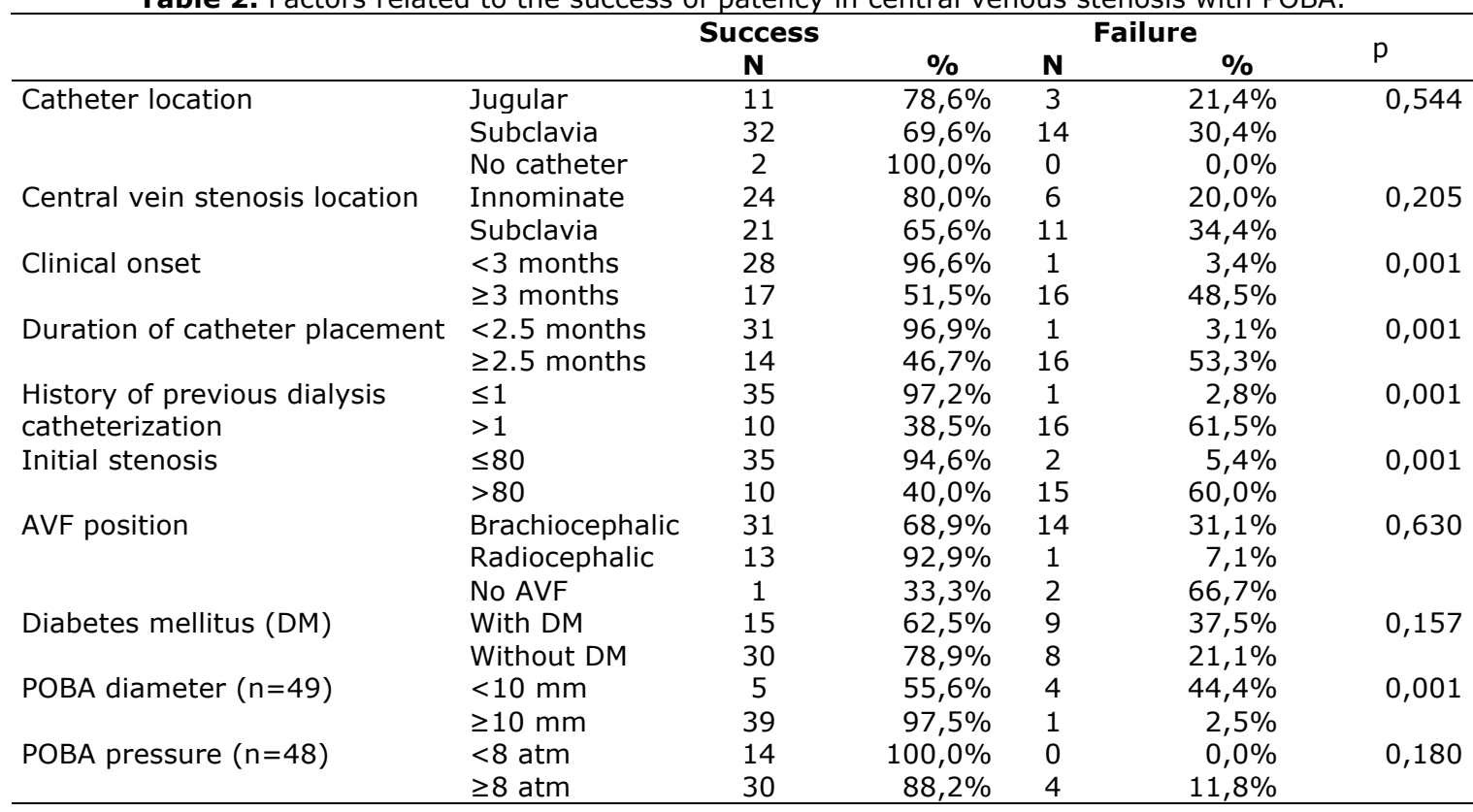

leads to the higher occurrence of trauma on the venous wall. This trauma causes an inflammatory process, thrombus formation, and changes in venous wall histology. The characteristics data also showed clinical symptoms that occurred was $\geq 3$ months $(53.2 \%)$, and the duration of catheter placement was $<2.5$ months $(51.6 \%)$, indicating that screening was also not optimal. The most common symptom complained by the subjects in this study was arm edema $(85 \%)$, which swelled to the face $(77.5 \%)$, and access to hemodialysis that could not be used (44.7\%).

The first significant factor associated with the success of the endovascular procedure in patients with central venous stenosis was the onset of clinical symptoms $<3$ months. Following the pathogenesis of CVS, it is due to turbulence in blood flow that causes an inflammatory response and stimulation of intimal hyperplasia, which will continue progressively if no intervention was done faster. ${ }^{6,7}$ In this study, if the intervention had been performed faster, the success was higher.

Other factors were the duration of catheter placement ( $<2.5$ months) and the history of a previous installation of a dialysis catheter before. Repeated friction of the catheter against the venous wall and blood turbulence causes thickening of the venous wall and platelet deposits, which in turn causes the veins to lose vascular tone. With the longer duration of catheter placement, the higher the risk of CVS, and vice versa..$^{4,8}$ This was in line with Hernandez's study (1998), which states that CVS occurs more frequently in more prolonged usage, which was 49 days compared to 29 days. ${ }^{9}$ Histological study about central vein after catheter placement by Forauer (2003) et al. found that in short-term catheter use ( $<14$ days), local intima injury occurred with endothelial denudation and attached thrombus layers. Meanwhile, on long-term use of catheters (>90 days), smooth muscle cell proliferation was found, which causes thickening of the venous wall. The focal area of catheter placement in the venous wall consists of thrombus at various stages, collagen, and endothelial cells.

Initial stenosis (less than $80 \%$ ) and diameter of POBA (>10 mm) affected the success rate of endovascular procedure in patients with central venous stenosis. This was consistent with the theory of histopathological changes in central venous specimens, which showed directional atherectomy or occlusion, showing intimal hyperplasia, thrombus organization, endothelial cells, and increased collagen, possibly leading to the development of fibrin, and the presence of fibrotic tissue, in patients with symptomatic stenosis. The thicker the fibrosis $(>80 \%)$, the higher the venoplasty failure rates with POBA and the smaller the diameter size of the used POBA $(<10 \mathrm{~mm})$.

\section{CONCLUSION}

Some factors influence the success of POBA in CVS cases, which were the onset of clinical symptoms (<3 months), the duration of catheter placement (<2.5 months), history of previous catheter placement, initial stenosis $(\leq 80 \%)$, and diameter of POBA $(>10 \mathrm{~mm})$. A better screening strategy is needed to detect CVS cases so that the intervention could be performed faster.

\section{ACKNOWLEDGMENTS}

The author states the original work, and there is no conflict of interest in doing this research.

\section{ORCID ID OF AUTHORS}

Akhmadu Muradi

https://orcid.org/0000-0003-0165-9045

Donie Firdhianto

https://orcid.org/0000-0003-2632-3839

Aria Kekalih

https://orcid.org/0000-0001-7811-097X 


\section{REFERENCES}

1. Taal MW, Chesterton LJ, McIntyre CW. Venography at insertion of tunnelled internal jugular vein dialysis catheters reveals significant occult stenosis. Nephrol Dial Transplant.2004;19(6):1542-5.

2. Agarwal AK. Central Vein Stenosis: Current Concepts. Adv Chronic Kidney Dis.2009;16(5):360-70.

3. Banshodani M, Kawanishi H, Shintaku S, Moriishi M, Yamashita T, Ago R, et al. Percutaneous transluminal angioplasty for central venous disease in dialysis patients: influence on cardiac function. J Vasc Access. 2014;15(6):492-7.

4. Thwaites SE, Robless $\mathrm{P}$ a. Central vein stenosis in an Asian hemodialysis population. Asian Cardiovasc Thorac Ann [Internet]. 2012;20(5):560-5.

5. Tapaneeyakorn J, Inman T, Panpikul T, Wedsart B, Suvikrom J. Vascular access stenosis and central vein obstruction interventions: Five years of experience in ramathibodi hospital. J Med Assoc Thail.2012;95(9):1211-8.

6. Barrett N, Spencer S, McIvor J, Brown EA. Subclavian stenosis: a major complication of subclavian dialysis catheters. Nephrol Dial Transplant [Internet]. 1988;3(4):423-5. Available from: http://www.ncbi.nlm.nih.gov/pubmed/3140128

7. Mickley V. Central Vein Obstruction in Vascular Access. Eur J Vasc Endovasc Surg.2006;32(4):439-44

8. Barrett, N ; Spencer, S; Mclovor, J ; Brown E. Subclavian stenosis: a major complication of subclavian dialysis catheters. Nephrol Dial Transpl. 1988;3(4):423-5.

9. Trerotola S. Hemodialysis Catheter Placement and Management. Radiology. 2000;215(3):651-8. 